\title{
Proceedings of the Optical Convention, I926.
}

THE exhibition of instruments associated with the Optical Convention having been described in our issue of April 24, while the very entertaining optical illusions arranged by Sir Richard Paget and Dr. R. S. Clay were fully noticed in the lay press, the present article will be devoted to a brief review of some of the papers read during the daily sessions, which occupied two lecture-theatres at the Imperial College of Science and Technology, South Kensington, throughout the week of the Convention. As there were nearly a hundred papers in all, it is impossible to refer here to more than a few of them, but it is understood that the Proceedings of the Convention, containing the complete text, will be published in time to be available at the Oxford meeting of the British Association.

The contribution of outstanding merit was undoubtedly Mr. J. Guild's " Survey of Modern Developments in Colorimetry," and the discussion of this and other topics was enhanced in interest by the presence of Mr. H. E. Ives, of the United States, to whose labours the science of optics is so much indebted. There are few subjects upon which textbook information lags so far behind the available knowledge as the investigation of colour, and it would be difficult to exaggerate the value of this report, which is not less remarkable for its lucidity than for the directness with which it selects for discussion topics which are of greatest practical interest. It was news to many who might be expected to know about such things that the photometry of colours has been put upon such a basis that "the luminosities of different coloured lights can be equated to one another, or added together, in exactly the same way as a gallon of oil can be equated, on a volumetric scale, to a gallon of water, and as the total volume of a gallon of oil added to a gallon of water will measure two gallons."

Various criteria for equality of brightness have been examined, and it has been found that the difficulty which is experienced in judging the equality in brightness of two dissimilarly coloured lights is due to adventitious psychological factors which can be eliminated by statistical methods. Simple photometry therefore provides the basis of the modern theory, but flicker photometry can also be used as a derivative method of comparison. The paper deals very thoroughly both with the underlying theory and with the practical methods of colour measurement and specification, and shows that the whole subject is now upon the level of an exact science: colour mixtures can be specified numerically with precision. The terminology is still somewhat confused, however, and the Optical Society, following the example of the corresponding American body, is seeking information by means of a questionnaire with the view of standardising the nomenclature. The author considers that the most pressing need of the science is a statistical standardisation of the chromatic properties of the normal eve: the laws of colour mixture are subject to fluctuations, being slightly different for different individuals, and systematic experiment is necessary to ascertain the norm. The importance of experimenting under standard conditions as to the size of the visual field, the method of illumination, etc., is also insisted upon.

Another interesting paper on this subject was that by Dr. W. Peddie, dealing with the effects of fatigue on the sensations produced by colour mixtures. The industrial aspect was brought out in a paper by Mr. Gamble, who put forward suggestions for the standardisation of coloured inks for three-colour printing, and one by Dr. S. G. Barker and Mr. H. R. Hirst on the work of the British Research Association for the Woollen and Worsted Industries with regard to the fading of colours.

The use of the photo-electric cell in photometry is an interesting modern development. Dr. N. R. Campbell, the originator of the idea, and Mr. M. K. Freeth described an investigation, carried out by this method, of the variations in the properties of tungsten filaments in vacuum lamps. The luminous efficiency of successive samples cut from a single filament is found to vary by several parts in a thousand, and the sensitivity of the method is well illustrated by the present study of this phenomenon. The colour temperatures of the samples are compared by means of a rubidium cell, covered by a filter so as to have nearly the same colour-sensitivity characteristic as the eye, and the electrical magnitudes concerned are carefully measured; an estimated accuracy of 0.05 per cent. is achieved, and it is found that more consistent results are obtained if lamps are rated by current instead of by voltage. Mr. T. H. Harrison has extended the method to the measurement of directional candle power, as distinct from the total flux from a lamp. The difficulties are considerable owing to the dissimilarity in the characteristics of individual cells and to fatigue effects, but it is claimed that by means of suitable precautions an accuracy better than $\mathrm{o} \cdot \mathrm{I}$ per cent. can be obtained.

Among the papers on ophthalmic subjects may be mentioned two, by Mr. O. Aves and Mr. W. Swaine respectively, dealing statistically with the distribution among the population of various defects in eyesight. These papers contain much information on such matters as the age and sex distribution of optical defects, the results obtained by correction, the occupations of the patients, etc. There is a tendency for women to seek the optician's aid at an earlier age than men, possibly on account of their being compelled to do needle-work : in each case the frequencyage curve shows two maxima, one in the early twenties and the other in the later forties. As regards the effect of occupation, sewing and kindred tasks claim by far the largest proportion of victims: half as many of the latter are clerks, and one quarter as many are teachers, students, etc. The subject appears to be a somewhat new one, and the authors express dissatisfaction with the material available. A standardised system of analysis also appears to be desirable.

The subject of optical glass is of great interest for those who are concerned as to the fate of the British optical industry. The matter is of wide importance ; the optical industry is intimately related to the security and well-being of the nation, for on this branch of the industry all the others are dependent. It is satisfactory to know that whereas before the War only one British firm was engaged in the manufacture, that number has now been trebled. Mr. W. H. Chance and Mr. W. M. Hampton gave an interesting account of the work of their firm, showing that the number of types of glass which it makes has increased from the pre-War figure of 26 to II 2 at the present day. Glass colour filters are made in place of the former gelatine filters : a third colour, distinguishable under all conditions from green and red, has been found for railway signals, enabling home signals to be discriminated from distant signals: 'Crookes' glass has been introduced for excluding ultra-violet radiation, and 'vitaglass' for admitting it. The 
latter has been fitted in the monkey-house at the Gardens of the Zoological Society at Regent's Park, and is said to have had a beneficial effect on the welfare of the inmates; but it seems doubtful whether the indiscriminate use by the public of lamps emitting strong ultra-violet radiation is in any degree safe.

In connexion with the subject of optical glass, considerable interest attaches to a paper by $\mathrm{Mr}$. W. D. Haigh describing a method for determining the absorption coefficient of glass for light passing through it. His results for British glass are confirmed by the experience of the National Physical Laboratory, and in view of this confirmation of the accuracy of the method, the author's comparison of the transparencies of British and foreign glasses is of some importance. The British glasses are on the whole considerably more transparent to light of the shorter wave-lengths, and are therefore more suitable for the construction of fast camera lenses. A paper by Lord Rayleigh was devoted to the difference in transparency between silica glasses made from sand and from rock crystal respectively, the comparative opacity of the former being attributable to the presence of bubbles.

A number of papers dealt with instrument design and manufacture, but as this aspect of the Convention has already been described in our columns, no further space need be devoted to it here, except as regards two particularly interesting papers on surveying instruments for the Colonies, by Mr. J. L. Rannie and Col. H. St. G. Winterbottom respectively. The first of these authors reviewed the requirements of theodolites for use in Canada, reduction in size and weight and increased speed of manipulation being the chief of these. The paper contains some valuable hints to British manufacturers, and suggests the use of aluminium alloys for the metal parts, elimination of counterpoises by suitable design, sacrifice of excessive accuracy, reduction of the number of micrometers, enlargement of the field of view to facilitate rough alignment, and other innovations. Mr. Rannie believes that definite changes along the lines which he suggests are necessary if Great Britain is to hold the Canadian market. Col. Winterbottom, on the other hand, gave an account of the experience of the Indian Survey which was somewhat more flattering to British manufacture: continental instruments fail to stand the rough conditions of transport to India, and the superior workmanship of British-made instruments is much in their favour. Indeed, it would appear from both these papers that British instruments suffer from the disadvantage (from a manufacturer's point of view) that they do not wear out. It is Col. Winterbottom's opinion that British-made instruments are the most economical in the long run, in spite of their initial cost.

C. W. H.

\section{The Sense Organs of Insects.}

I ITTLE is definitely known from the physiological point of view of many of the sense organs of insects. Except in the case of the eyes and the tactile hairs, it is largely a matter of conjecture to classify them according to function. It is desirable, therefore, to group the various types of these organs into different categories based upon their essential structure.

Most of the sense organs of insects can be classified according to the form of their external cuticular components, as was carried out by Schenk in 1903 . Dr. R. E. Snodgrass, in a recent valuable contribution entitled "The Morphology of Insect Sense Organs and the Sensory Nervous System" (Smithsonian Misc. Coll. 77, No. 8, 1926), follows in the main this same general classification. His paper commends itself to all interested in the subject, since he has brought together the chief results of the great mass of recent German work in a convenient form. Dr. Snodgrass points out that, since no sensory cytons have been found in the central ganglia of insects, we have to conclude that they are represented by the sensory cells found in relation with the body-wall and alimentary canal. This fundamental conclusion contrasts with what is known concerning the sensory cytons of the vertebrate nervous system, where they are located in the spinal ganglia. Furthermore, the origin and growth of the sensory nerves have not so far been traced in any insects. All we do know is that the sensory nerves of those animals terminate in the central ganglia in finely branching nerve fibrils which constitute the sensory neuropiles.

The simplest type of insect sense organ or sensillum is little more than a slender hair. Histologically it consists of a large hair-forming cell, a membrane cell, which secretes the membrane uniting the hair to its socket, and a sense cell. The latter lies beneath the hypodermis and sends out a nerve process to the base of the hair. It seems reasonable to conclude that such tactile hairs were the first specific sense organs to be acquired by insects, and that from them were developed organs for perceiving chemical stimuli, sound stimuli, or whatever other stimuli are perceptible to insects. In the case of the eyes, the fundamental elements in all varieties of these organs among insects are photoreceptive cells of hypodermal origin. These cells correspond with the sense cells of other sense organs and may be termed the sense cells of the ocular sensillum. Associated with them are cells of hypodermal origin which differ greatly from the enveloping cells of the other sense organs. This fact leads Dr. Snodgrass to conclude that any theory which would derive an ocular sensillum from that of an original sensory hair, as was suggested by Patten in 1890 , is too far-fetched to be convincing.

The cuticular part of a sense organ is adapted for the reception of a specific stimulus. In the case of a chemoreceptor the organ is somehow penetrable by odour or taste substances. The idea that such organs were perforated to allow of the substances to be perceived to come in contact with nerve-endings is not upheld. The cuticular walls of these sense organs are, in many cases, not more than half a micron in thickness, and it is reasonable to suppose that they allow of the passage of odour or taste substances. In support of this view Dr. Snodgrass quotes the observation of Vogel, who noted that the membranous cupola of a basiconic sensillum of the wasp is coloured by haematoxylin, and hence is permeable by it. There is no doubt that a better acquaintance with the physiology, the senses, and the tropisms of insects will provide an insight into how these creatures so successfully maintain their dominant place in Nature, notwithstanding man's most energetic efforts to repress them.
A. D. I.

NO. 2948, VOL. II 7$]$ 